\title{
Discriminating from species of Curcumae Radix (Yujin) by a UHPLC/Q-TOFMS-based metabolomics approach
}

Fang Liư ${ }^{1}$ Xu Bai ${ }^{2}$, Feng-Qing Yang ${ }^{3}$, Xiao-Jing Zhang ${ }^{1}$, Yuanjia Hu ${ }^{1}$, Peng Li ${ }^{1}$ and Jian-Bo Wan ${ }^{\text {* }}$

\begin{abstract}
Background: Chinese medicinal herbs may use more than one species of Curcumae Radix (Yujin) is the tuberous roots of Curcumae wenyujin, C. kwangsiensis, C. phaeocaulis and C. longa. This study aimed to characterize the chemical profiles of these different species of Curcumae Radix, and develop a method for rapid discrimination of these species by ultra-high performance liquid chromatography-quadrupole time-of-flight mass spectrometry (UHPLC/Q-TOFMS) combined with multivariate statistical analysis.

Methods: The metabolomes of 33 different batches of Curcumae Radix derived from four Curcumae species were profiled by UHPLC/Q-TOFMS. The resulting sample codes, $t_{R}-m / z$ pairs and ion intensities were processed by unsupervised principal component analysis (PCA) and supervised orthogonal partial least squared discriminant analysis (OPLS-DA) to characterize the chemical composition of Curcumae Radix across the four different species.

Results: Obvious differences were observed in the chemical compositions of the Curcumae Radix samples derived from the four different species according to PCA and OPLS-DA. These results suggested that curcumin, curcumenone, curcumenol and zederone could be used as unique chemical markers for C. longa, C. wenyujin, C. phaeocaulis and C. kwangsiensis, respectively.

Conclusions: This study developed a UHPLC/Q-TOFMS method coupled with multivariate statistical analysis to discriminate between Curcumae Radix samples from four different Curcumae species, i.e., C. Ionga, C. wenyujin, C. phaeocaulis and C. kwangsiensis. Notably, this new approach resulted in the identification of curcumin (a), curcumenone (b), curcumenol (c) and zederone (d) as unique chemical markers for the identification.
\end{abstract}

\section{Background}

Herbal medicines may be derived from specific single or multiple plant species, and a herb may have more than one species. The increasing popularity of multi-species herbs in medical use was documented in the 2010 edition of the Chinese Pharmacopoeia [1]. For instance, snow lotus herb (Xuelian), which has several health benefits, is mainly derived from Saussurea involucrata, S. laniceps and S. medusa [2], whereas dragon's blood (Xuejie), which is used as a medicine, is mainly derived

\footnotetext{
*Correspondence: jbwan@umac.mo

${ }^{1}$ State Key Laboratory of Quality Research in Chinese Medicine, Institute of Chinese Medical Sciences, University of Macau, Macao, People's Republic of China

Full list of author information is available at the end of the article
}

from Daemonorops draco and Dracaena cochinchinensis [3]. Previous studies have demonstrated that the chemical characteristics of multi-species herbal medicines can vary considerably depending on the originating species [3-5]. However, despite these findings, multi-species herbs are still treated as though they are identical. Each species of herb has its own genetic traits and geographical origins, which manifest themselves as unique primary and secondary metabolite patterns (i.e., metabolome) [3-5].

Curcumae Radix (Yujin) is used to treat a variety of different diseases, including hepatitis, cholecystitis, hyperlipidaemia and cancer [6-8]. Essential oils and curcuminoids are considered to be the major bioactive ingredients of Curcumae Radix [9], which is obtained 
from the dried tuberous roots of four Curcuma species, including Curcuma wenyujin, C. kwangsiensis, C. phaeocaulis and C. longa, as officially described in the Chinese Pharmacopoeia [10]. Given that Curcumae Radix can be derived from four different Curcuma species, there could be considerable differences in the chemical composition among these four species, which differences could affect their therapeutic effects. It is difficult to distinguish between the origins of raw materials in the clinic because of their similar morphological features. To date, several analytical methods, including liquid chromatographymass spectrometry (LC-MS) [11, 12], high-performance liquid chromatography (HPLC) [13, 14], gas chromatography-mass spectrometry (GC-MS) $[15,16]$, thin layer chromatography (TLC) [17] and capillary electrophoresis (CE) [18], have been developed to discriminate among the different species of Curcuma based on the chemical diversity of several main ingredients, particularly sesquiterpenoids. Metabolomics could be readily used to qualitatively and quantitatively monitor variations in the chemical component profiles of herbal medicines derived from different species $[19,20]$, as well as those subjected to different processing methods [21,22], cultivation times [23] and geographical locations [24]. Recently, a GC-MSbased metabolomics method was successfully established to discriminate three Curcuma species according to the global chemical difference in Curcuma rhizomes (Ezhu) [19]. However, several sesquiterpene compounds from Curcuma plants are heat-sensitive. For example, furanodiene degrades to curzerene via a [3,3]-sigmatropic reaction (Cope rearrangement) upon heat treatment [25], whereas $(4 S, 5 S)$-germacrone-4,5-epoxide cyclizes through a transannular reaction on exposure to heat [26].

The aim of this study was to characterize the chemical profiles of Curcumae Radix and develop a rapid method to discriminate between the species by ultra-high-performance liquid chromatography-quadrupole time-of-flight mass spectrometry (UHPLC/Q-TOFMS) combined with multivariate statistical analysis.

\section{Methods}

\section{Plant materials and chemical reagents}

Thirty-three batches of authentic Curcumae Radix were collected from Good Agricultural Practice (GAP)-certified farms located in Rui'an and Leqing, Zhejiang province (C. wenyujin, $\mathrm{n}=12$ ), Yulin and Linshan, Guangxi province (C. kwangsiensis, $\mathrm{n}=6$ ), and Leshan and Chengdu, Sichuan province (C. phaeocaulis, $\mathrm{n}=6$; and C. longa, $\mathrm{n}=9$ ) provinces of China during December, 2012. The botanical origins of the different Curcumae Radix samples were morphologically recorded [27] based on the whole plants when they were collected from the GAP-certified farms, and were subsequently identified by
Dr. Fengqing Yang at the School of Chemistry and Chemical Engineering, Chongqing University, China. Voucher specimens of the Curcumae Radix samples from four different Curcuma species (No. YJ01-YJ33) were deposited at the Institute of Chinese Medical Sciences, University of Macau, Macao, China. HPLC-grade methanol and acetonitrile were purchased from Baker Company (Sanford, ME, USA). LC-MS grade formic acid was purchased from Sigma-Aldrich (St. Louis, MO, USA). All of the other chemicals and solvents used in the current study were purchased as the analytical grades and used as received. Ultra-high-purity water was prepared using a Millipore SAS-67120 (Molsheim, Cedex, France).

\section{Pressurized liquid extractions (PLE)}

Samples for analysis were prepared using a Dionex ASE 200 system (Dionex, Sunnyvale, CA, USA) according to a previously described procedure [28]. Briefly, Curcumae Radix was dried at $60{ }^{\circ} \mathrm{C}$ for $2 \mathrm{~h}$, and then pulverized in a mill to give a homogeneous powder (60 mesh). A portion of the dried powder (approximately $0.5 \mathrm{~g}$ ) was mixed with diatomaceous earth $(0.5 \mathrm{~g})$, and the resulting mixture was transferred to an $11-\mathrm{mL}$ stainless steel extraction cell. The sample was subsequently extracted under the following conditions: solvent, methanol; pressure, $6.89 \times 10^{3} \mathrm{kPa}(1000 \mathrm{psi})$; temperature, $100{ }^{\circ} \mathrm{C}$; static extraction time, $5 \mathrm{~min}$; number of extractions, 1; and flush volume, $40 \%$. After the extraction, the PLE extract was transferred to a $25-\mathrm{mL}$ volumetric flask, which was made up to its volume with methanol. An aliquot of the extract was centrifuged (Heraeus Multifuge; Thermo Fisher Scientific, USA) at $11,200 \times g$ for $5 \mathrm{~min}$, and the supernatant was filtered through a $0.22-\mu \mathrm{m}$ polytetrafluoroethylene filter (Whatman, NJ, USA) prior to being injected into the UHPLC/Q-TOFMS system.

\section{LC-MS analysis}

UHPLC analysis was performed by an ACQUITY UHPLC system (Waters, Milford, MA, USA) equipped with a binary solvent delivery manager, auto-sampler and high temperature (HT) column oven. The UHPLC system was also equipped with an ACQUITY HSS T3 $\mathrm{C}_{18}$ column $(100 \times 2.1 \mathrm{~mm}$ i.d., $1.8 \mu \mathrm{m}$; waters $)$ for the chromatographic separation of the sample mixture. The column was eluted with a binary gradient elution system consisting of (solvent A) acetonitrile and (solvent B) $0.1 \%$ aqueous formic acid solution (containing $1 \%$ acetonitrile). Chromatographic separation was achieved under the following elution conditions: isocratic $1 \%$ solvent A (0-0.5 min); linear gradient from $1 \%$ to $99 \%$ solvent A (0.5-5 min); isocratic $99 \%$ solvent A (5.0-6.0 min); and linear gradient from $99 \%$ to $1 \%$ solvent A $(6.0-8.0 \mathrm{~min})$. The flow rate and column temperature were set at 
$0.45 \mathrm{~mL} / \mathrm{min}$ and $45^{\circ} \mathrm{C}$, respectively. An aliquot $(5 \mu \mathrm{L})$ of each sample was injected into the column.

The UHPLC system was connected to a Xevo G2 quadrupole time-of-flight (Q-TOF) mass spectrometer (waters) with an electrospray ionization (ESI) source. Samples of the eluent from the UHPLC system were analyzed on this system under the following conditions: ionization mode, positive; source temperature, $120{ }^{\circ} \mathrm{C}$; capillary voltage, $3000 \mathrm{~V}$; sampling cone voltage, $30 \mathrm{~V}$; extraction cone voltage, $4 \mathrm{~V}$; cone gas flow, $20 \mathrm{~L} / \mathrm{h}$; desolvation gas flow, $800 \mathrm{~L} / \mathrm{h}$; and desolvation temperature, $450{ }^{\circ} \mathrm{C}$. Mass data were collected in the range of $\mathrm{m} / \mathrm{z}$ $50-1200 \mathrm{Da}$ in the centroided mode. A lock-mass calibrant of leucine-enkephalin was continuously introduced to the mass spectrometer at a concentration of $200 \mathrm{ng} /$ $\mathrm{mL}$ via a lock-spray interface at a flow-rate of $50 \mu \mathrm{L} / \mathrm{min}$. This process generated a reference ion in the positive ionization mode (i.e., $[\mathrm{M}+\mathrm{H}]^{+}=556.2771$ ), which ensures a high level of accuracy during the MS analysis.

\section{Method validation}

A pooled sample of Curcumae Radix extracts was prepared by mixing $200 \mu \mathrm{L}$ of each batch of Curcumae Radix extract in a single sample vial. The resulting mixture was used to provide a representative quality control (QC) sample containing all of the analytes at an average concentration. The QC sample was analyzed six times at the beginning of each run to ensure that the system was properly equilibrated. The QC sample was also analyzed after every three tested samples to further monitor the stability of the analytical process based on three representative ions in the chromatogram (i.e., $t_{\mathrm{R}} 2.50 \mathrm{~min}, \mathrm{~m} / \mathrm{z}$ $312.1601 ; t_{\mathrm{R}} 4.16 \mathrm{~min}, \mathrm{~m} / z$ 219.1744; and $t_{\mathrm{R}} 4.80 \mathrm{~min}$, $m / z 231.1385$ ), which were selected for the validation of the method in the positive ionization mode. The relative standard deviations (RSDs) of peak intensity, retention time and molecular weight for the selected ions in the pooled QC samples were calculated $(\mathrm{RSD}=\mathrm{SD} /$ mean)] to evaluate the reproducibility and stability of the method.

\section{Data processing and pattern recognition}

The raw UHPLC/Q-TOFMS data were imported to the Progenesis QI software (Waters) for peak detection and alignment using the following parameters: mass tolerance within $5 \mathrm{ppm}$; retention time tolerance within $0.3 \mathrm{~min}$; and relative mass error of theoretical fragmentation within $5 \mathrm{ppm}$. All data were normalized to the summed total ion intensity per chromatogram and threedimensional data matrices were generated consisting of variable ID numbers (retention time- $m / z$ value), sample codes and normalized peak areas. These matrices were then entered into the SIMCA-P 13.0 software (Umetrics
$A B$, Sweden) for multivariate statistical analysis, including unsupervised principal component analysis (PCA). The resulting PCA model was evaluated and interpreted in terms of the $R^{2} X$ (cum) and $Q^{2}$ (cum) values in its score plot. The $R^{2} X$ value represents the explanatory capacity of the variables, whereas $Q^{2}$ provides an indication of the predictive capability of the model. The $R^{2} X$ and $Q^{2}$ values were both found to be close to 1.0 , indicating the good fitness of this method. Various chemical components were extracted from the loading plot of the PCA and a scatter plot (S-plot) of OPLS-DA as being responsible for the difference between the Curcuma species. Furthermore, ions with high variable importance on projection (VIP) values from the $S$-plots were considered to be potential chemical biomarkers, and subsequently subjected to further structural identification. A one-way ANOVA was also employed to test the significance of any differences observed between these markers among the four different species of Curcuma Radix. Differences with $P \leq 0.05$ were considered statistically significant.

\section{Results and discussion \\ Method development and validation}

It can be difficult to distinguish between the different types of compound found in the different species of Curcumae Radix (e.g., the essential oils and curcuminoids) using the short analytic times associated with UHPLC. With this in mind, we conducted a small pilot study to optimize the chromatographic conditions used for the UHPLC analysis, including the column type and mobile phase, to achieve the highest possible resolution with the fastest separation time. Three ACQUITY UHPLC columns were evaluated in the current study, including BEH C18 $(100 \times 2.1 \mathrm{~mm}$ i.d., $1.7 \mu \mathrm{m})$, BEH HILIC $(50 \times 2.1 \mathrm{~mm}$ i.d., $1.7 \mu \mathrm{m})$ and HSS T3 C18 $(100 \times 2.1 \mathrm{~mm}$ i.d., $1.8 \mu \mathrm{m})$ columns. Among these columns, the ACQUITY HSS T3 C18 column was found to be the most suitable for the analysis of Curcumae Radix because it afforded good peak capacity and the best resolution of the major components. A variety of different mobile phases were evaluated for the elution of the column, including acetonitrile-water and methanol-water systems. These mobile phase systems were also evaluated in the presence of various modifiers, and the results were compared to determine which system gave the best resolution. The results of this screening process revealed that a combination of acetonitrile (organic mobile phase) and $0.1 \%$ aqueous formic acid (containing $1 \%$ acetonitrile; aqueous mobile phase) was optimal for the simultaneous separation of the major components in Curcumae Radix, as well as being compatible with the MS analysis (i.e., no ionization suppression from the formic acid). The addition of $1 \%$ acetonitrile to the aqueous phase was found 
to be beneficial for stabilizing the baseline drift of instrument analysis. Ionization was conducted in the positive and negative ionizations modes to achieve maximum signal strength. It is noteworthy that higher levels of sensitivity and more structural information were obtained in the positive ionization mode. Having established the optimized chromatographic and MS conditions, we were able to successfully separate and identify the major chemical components in Curcumae Radix within 8 min (Fig. 1). To validate this method, we measured the RSD of the peak areas and $m / z$ values (i.e., $[\mathrm{M}+\mathrm{H}]^{+}$) of selected peaks (i.e., $t_{\mathrm{R}} 2.50 \mathrm{~min}, \mathrm{~m} / z 312.1601 ; t_{\mathrm{R}} 4.16 \mathrm{~min}, \mathrm{~m} / z 219.1744$; and $t_{\mathrm{R}} 4.80 \mathrm{~min}, \mathrm{~m} / z$ 231.1385), which were less than 5.46 and $0.01 \%$, respectively. Furthermore, the retention times of these peaks remained exactly the same over 16 runs, which indicated that the established method was robust with good reproducibility and stability for the analysis of experimental Curcumae Radix samples.

\section{Multivariate statistical analysis}

Visual inspection of the results revealed that there were obvious differences among the four different Curcumae species. However, it was not possible to differentiate between these four groups based on visual inspection alone. We therefore applied multivariate statistical analysis to intuitively refine intergroup differences in the chemical components and identify potential chemical biomarkers that could be used to distinguish between the different groups.

The resulting dataset contained sample code, $t_{\mathrm{R}}-m / z$ pair and ion intensity data, which were processed by PCA to clearly discriminate between the four species based on the differences in their chemical compositions. It is noteworthy that a total of $2599 t_{\mathrm{R}}-m / z$ pairs were extracted in the positive ion mode for all of the samples analyzed in this study. After Pareto scaling and mean-centering, these data were displayed as scores and loadings in a coordinate system of principal components resulting from data dimensionality reduction. The three-component PCA score plots (Fig. 2A) showed that the 33 Curcumae Radix samples could be clearly classified into four different clusters depending on their species, according to the differences in their global chemical profiles. Although the clusters corresponding to C. wenyujin and C. phaeocaulis were close to each other, they could be clearly distinguished by PC2. Sevenfold cross validation was used to assess the validity of the model. Notably, all of the observations in the current study fell within the Hotelling T2 (0.95) ellipse. Furthermore, the $R^{2} X$ (cum) and $Q^{2}$ (cum) values were determined to be 0.788 and 0.724 , respectively, thereby highlighting the quality of our PCA model.

\section{Characterization of chemical markers}

A loading plot of the PCA was constructed to identify the characteristic chemical components responsible for the differences observed across the four species of Curcumae Radix. Each point in the loading plot represents a chemical component (variable), and the further each variable moves away from the main cluster of the analyzed chemicals, the more likely it is that this component makes a significant contribution to the intergroup differences. As shown in Fig. 2B, the loading plot of the PCA revealed that seven ions, including a $\left(t_{\mathrm{R}} 4.11 \mathrm{~min}, \mathrm{~m} / z\right.$ $369.1331), \mathbf{b}\left(t_{\mathrm{R}} 4.24 \mathrm{~min}, \mathrm{~m} / z 235.1696\right)$, c $\left(t_{\mathrm{R}} 4.31 \mathrm{~min}\right.$, $\mathrm{m} / z$ 235.1697), d $\left(t_{\mathrm{R}} 4.35 \mathrm{~min}, \mathrm{~m} / z 247.1333\right)$, e $\left(t_{\mathrm{R}}\right.$ $4.48 \mathrm{~min}, \mathrm{~m} / z 237.1851)$, f $\left(t_{\mathrm{R}} 4.58 \mathrm{~min}, \mathrm{~m} / z 237.1853\right)$ and $\mathbf{g}\left(t_{\mathrm{R}} 4.80 \mathrm{~min}, m / z 231.1383\right)$, were making significant contributions to the clusters. These ions were therefore considered to be potential chemical markers for discriminating between the four species of Curcumae Radix.

An $S$-plot of the OPLS-DA was constructed based on a comparison of the results for one species with those of the remaining three species to confirm the identities of the chemical markers obtained from the loading plots, as well as identifying the characteristic chemicals of each species (Fig. 3). In this particular case, we used $C$. wenyujin as an example. The 12 Curcumae Radix samples derived from $C$. wenyujin were defined as groups, whilst the remaining 21 samples were marked as the second group, which formed the basis of our OPLS-DA model. The resulting $S$-plot is shown in Fig. 3A, where each point indicates an ion $t_{\mathrm{R}}-\mathrm{m} / z$ pair. The $\mathrm{X}$ and $\mathrm{Y}$ axes represent the contribution and confidence of the variables, respectively. In this plot, the further a specific data point is from zero on the $\mathrm{X}$ or the $\mathrm{Y}$ axis, the greater its contribution or confidence level for the two-group separation, respectively. The points at either ends of the S-shaped curve therefore represent potential chemical markers with the highest confidence [29]. In terms of the results, three ions (b, e and $\mathbf{f}$ ) were found at the end of the S-shaped curve in the bottom-left corner of the graph, which were considered to be characteristic components that made the greatest contribution to distinguish C. wenyujin from the other three species. These components were responsible for the variance observed in the Curcumae Radix derived from $C$. wenyujin versus the other three species. Two ions $(\mathbf{d}, \mathbf{g})$ were found to be characteristic markers capable of discriminating $C$. $k$ wangsiensis from the other species of Curcumae Radix (Fig. 3B). In contrast, ions a and $\mathbf{c}$ were found to be specific markers for the Curcumae Radix derived from $C$. longa and C. kwangsiensis, respectively. 


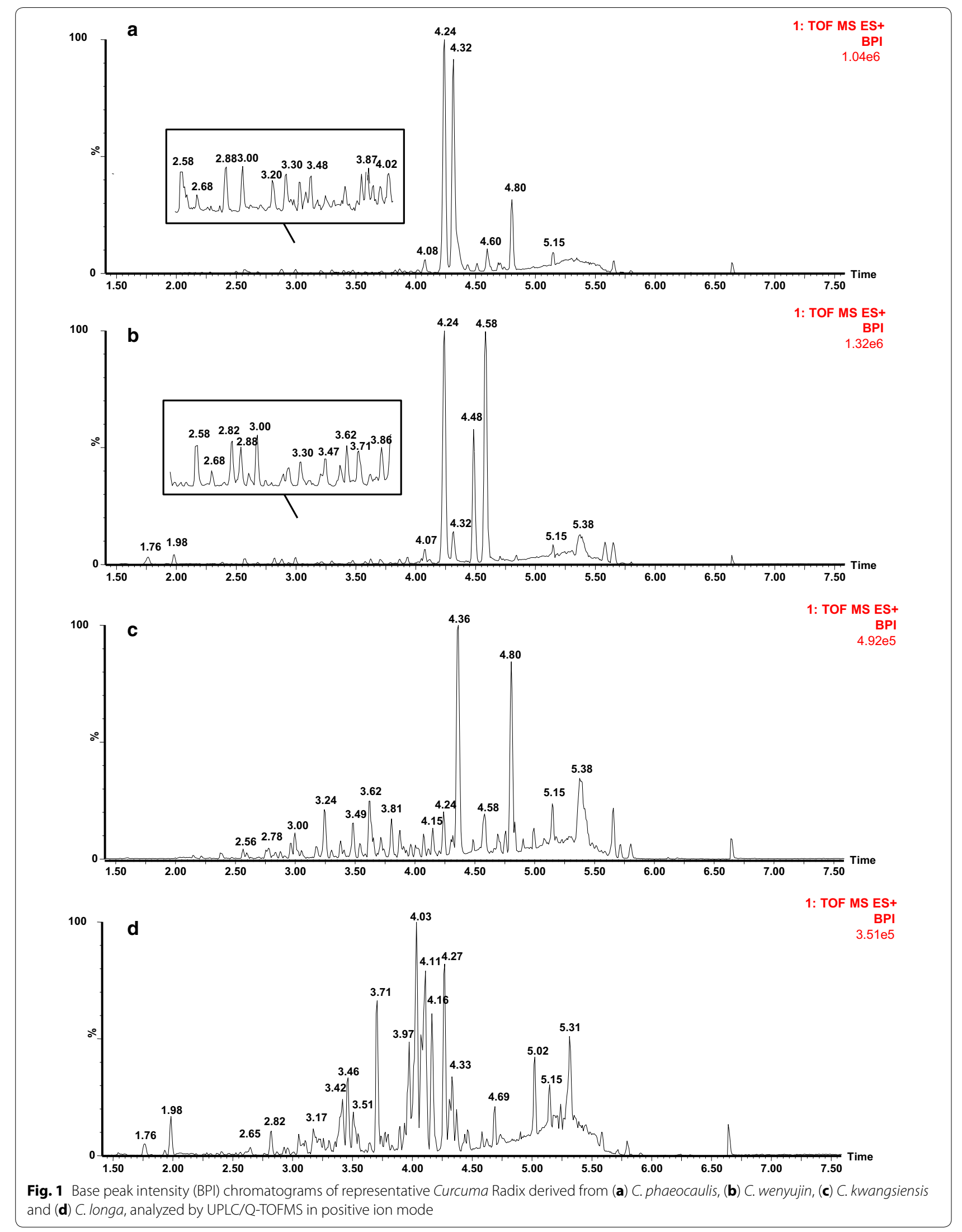



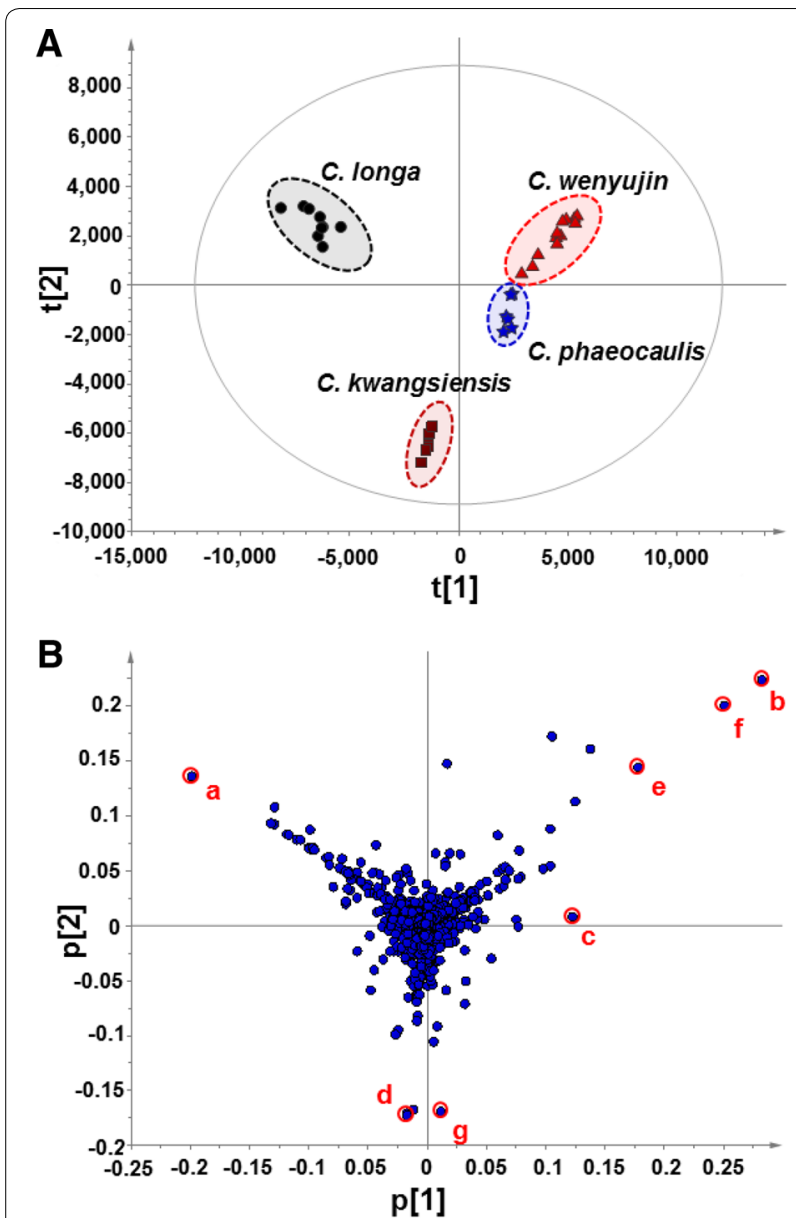

Fig. 2 PCA/Scores plot $(\mathbf{A})$ and loading plot (B) based on the global chemical profiling of Curcuma Radix derived from four species. The components contributing most to the differences were marked as a red circle, including (a) $t_{R} 4.11 \mathrm{~min}, \mathrm{~m} / \mathrm{z} 369.1331$; (b) $t_{\mathrm{R}} 4.24 \mathrm{~min}, \mathrm{~m} / \mathrm{z}$ 235.1696; (c) $t_{\mathrm{R}} 4.31 \mathrm{~min}, \mathrm{~m} / \mathrm{z} 235.1697 ;$ (d) $t_{\mathrm{R}} 4.35 \mathrm{~min}, \mathrm{~m} / \mathrm{z} 247.1333$; (e) $t_{\mathrm{R}} 4.48 \mathrm{~min}, \mathrm{~m} / \mathrm{z} 237.1851 ;(f) t_{\mathrm{R}} 4.58 \mathrm{~min}, \mathrm{~m} / \mathrm{z} 237.1853 ;$ and $(g) t_{\mathrm{R}}$ $4.80 \mathrm{~min}, \mathrm{~m} / \mathrm{z} 231.1383$

\section{Identification of chemical markers and their changes in relative intensities}

The Mass Fragment ${ }^{\mathrm{TM}}$ software package from waters was used to elucidate the structures of the different chemical components based on their fragmentation patterns. In this study, we were only able to identify seven chemical markers using the loading plots and the $S$-plots (Fig. 4). Furthermore, these structures were only tentatively assigned based on a comparison of their molecular weights, molecular formulas and MS/MS fragment ions with those published in the literature for compounds [11-16] isolated from Curcuma species, as well a comparison with known biochemical databases, such as MassBank (http://www.massbank.jp/), PubChem (http://pubchem.ncbi.nlm.nih.gov/), and Respect for
Phytochemicals (http://spectra.psc.riken.jp/). The error in the measured molecular mass values was determined to be less than $2 \mathrm{mDa}$ based on a comparison with the theoretical exact mass values provided by the authoritative websites mentioned above. Representative MS/MS spectra, chemical structures and proposed fragmentation pathway details for chemical marker a are shown in Fig. 4a. MS analysis of chemical marker a revealed an $\mathrm{m} / \mathrm{z}$ value of 369.1340 for $[\mathrm{M}+\mathrm{H}]^{+}$in the positive ionization mode, which suggested that its empirical molecular formula was $\mathrm{C}_{21} \mathrm{H}_{20} \mathrm{O}_{6}$. The differences in the mass of this parent ion and its two main fragment ions $(\mathrm{m} / z 285.1137$ and 177.0555) were 80 and $192 \mathrm{Da}$, which corresponded to loss of the $\mathrm{C}_{4} \mathrm{H}_{4} \mathrm{O}_{2}$ and $\mathrm{C}_{11} \mathrm{H}_{12} \mathrm{O}_{3}$, respectively. These data suggested that this compound was curcumin, based on a comparison with data from the literature [12, 30]. The most plausible interpretation of this fragmentation pathway is shown in Fig. 4c, which is primarily based on the information from the MS/MS data. Similarly, MS analysis of ion b (Fig. 4b) revealed an $\mathrm{m} / z$ value of 235.1699 for $[\mathrm{M}+\mathrm{H}]^{+}$in the positive ionization mode, which suggested that this compound had an empirical molecular formula of $\mathrm{C}_{15} \mathrm{H}_{23} \mathrm{O}_{2}$. Ion $\mathbf{b}$ gave a fragment ion with an $m / z$ value of 177.1273 , representing a $58 \mathrm{Da}$ mass difference compared with the parent ion, indicating the loss of $\mathrm{C}_{3} \mathrm{H}_{6} \mathrm{O}$. This daughter ion $(\mathrm{m} / z$ 177.1273) afforded four main fragment ions with $m / z$ values of 161.0966, 133.1015, 105.0861 and 91.0548, representing mass differences of 16, 44, 58 and $62 \mathrm{Da}$, respectively, which corresponded to the successive loss of the $\mathrm{CH}_{4}$, $\mathrm{CO}, \mathrm{CH}_{2}$ and $\mathrm{CH}_{2}$. These data therefore suggested that this compound was curcumenone and its fragmentation pathway is shown in Fig. 4d [31]. Based on the method described above, markers $\mathbf{c}-\mathbf{g}$ were tentatively identified as curcumenol, zederone, neocurdione, curdione and curzerenone, respectively, by comparing their data with those from the literature [31, 32], as well as searching the biochemical databases listed above (Additional file 1).

The signal intensities of the chemical markers identified in the different Curcumae Radix samples were analyzed by one-way ANOVA to provide a further comparison of the differences among the four species of Curcumae Radix. The marker levels varied considerably among the different Curcumae Radix samples (Fig. 5). The intensity of curcumin was found to be significantly $(P<0.0001)$ higher in $C$. longa than it was in any of the other three species (Fig. 5a), suggesting that curcumin could be used as a specific marker for distinguishing $C$. longa from the other three species. C. wenyujin showed significantly higher levels $(P<0.001)$ of curcumenone $(\mathbf{b})$, neocurdione (e) and curdione (f) than the other three species of Curcumae Radix (Fig. 5b, e and f). Furthermore, $C$. wenyujin contained higher levels of neocurdione and 


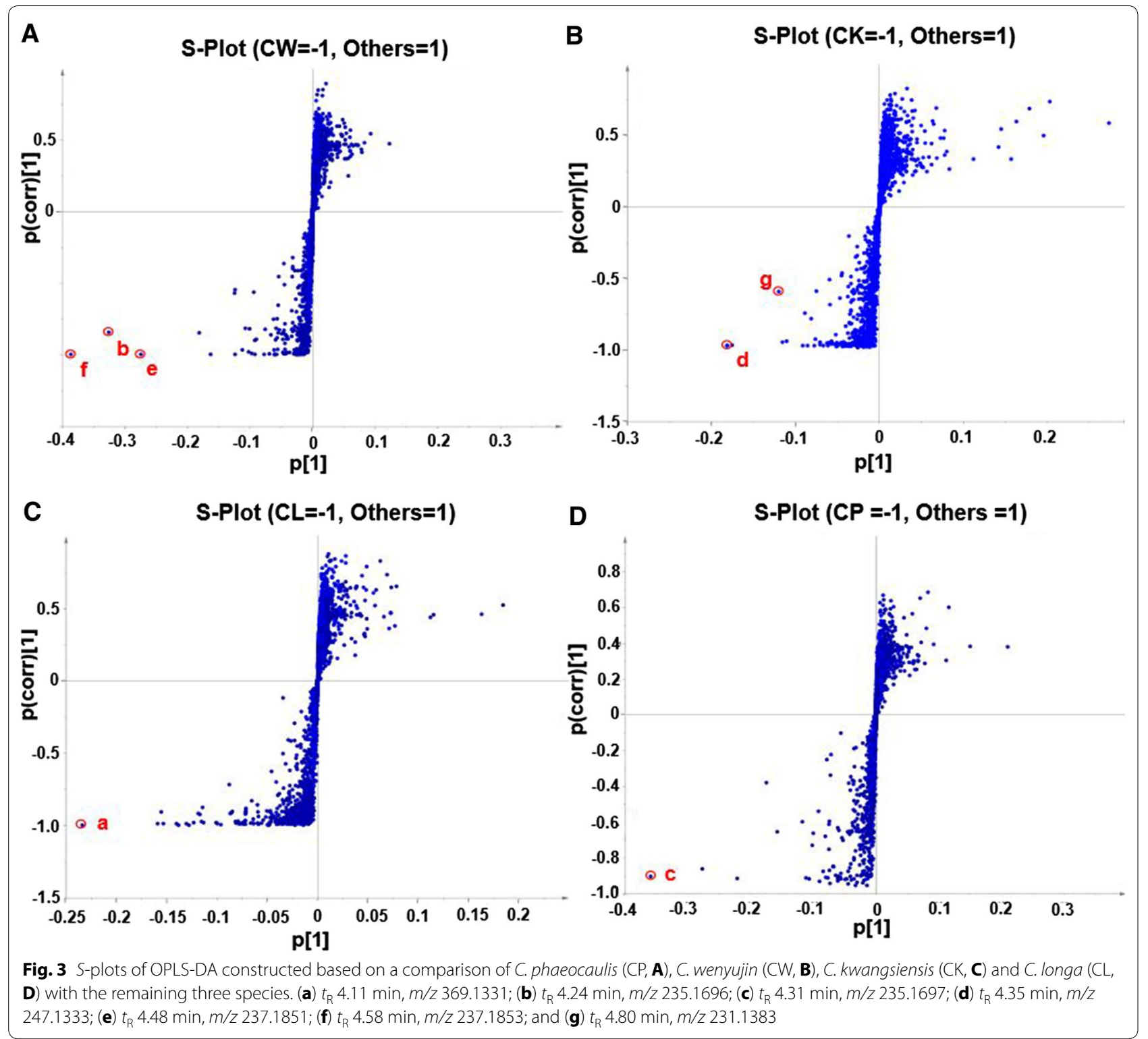

curdione (35- to 140-times higher) than any of the other three species of Curcumae Radix, suggesting that these compounds could be considered as markers for distinguishing $C$. wenyujin from the other three species. Curcuminoids and sesquiterpenoids are considered to be the main bioactive ingredients of Curcumae Radix [13, 14]. Curcuminoids, such as curcumin, have been reported to exhibit a wide range of interesting pharmacological activities, including antioxidative, anti-inflammatory, hypocholesterolemic, antihepatotoxic and anticancer effects [4]. Sesquiterpenoids, such as curcumenone, curdione and curzerenone, are the main components of the essential oils derived from Curcumae Radix, which have been reported to exhibit antioxidative, anti-tumor and antiviral activities [32].

\section{PCA analysis based on the identified chemical markers}

PCA was performed based on the seven chemical markers identified above to assess their ability to actively discriminate between the different species of Curcumae Radix. After normalizing the fused data, a 33 (objects) $\times 7$ (variables) dataset was constructed and subjected to PCA. The resulting PCA bi-plot is shown in Fig. 6a, where the observations and variables of the multivariate data are represented in the same plot. These data showed that variables with similar loadings 

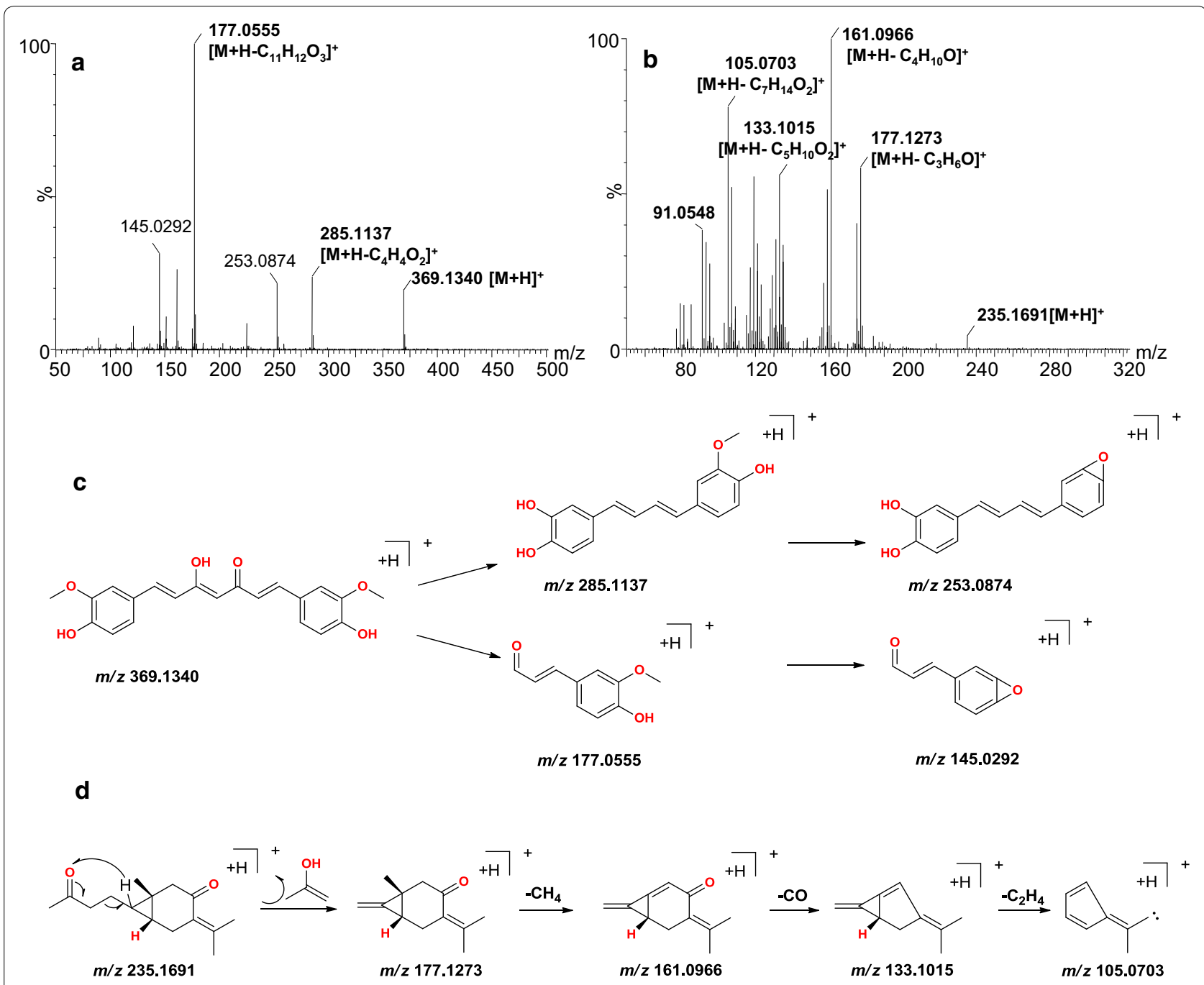

Fig. 4 Mass spectra for the proposed fragmentation pathways of curcumin $(\mathbf{a}, \mathbf{c})$ and curcumenone $(\mathbf{b}$, d $)$ in the positive ionization mode

appeared to be strongly correlated with the objects. In a similar manner to Fig. 2A, all of the Curcumae Radix samples were successfully separated into their correct species based on the differences in these seven chemical markers.

Based on the VIP values obtained from the $S$-plots of the OPLS-DA models (Fig. 3), curcumin (a), curcumenone (b), curcumenol (c) and zederone (d) were selected as unique markers for $C$. longa, $C$. wenyujin, $C$. phaeocaulis and C. kwangsiensis, respectively. PCA was also conducted based on these four unique markers using a 33 (objects) $\times 4$ (variables) data matrix, and the resulting PCA bi-plot revealed that all of the Curcumae Radix samples evaluated in the current study were successfully separated into the correct species based on these four markers. It is noteworthy that the separation observed between C. kwangsiensis and C. longa (Fig. 6B) was much clearer for the four chemical markers than it was for the PCA bi-plot constructed using the seven chemical markers described above (Fig. 6A). Our data therefore suggest that curcumin (a), curcumenone (b), curcumenol (c) and zederone (d) are unique chemical markers for C. longa, C. wenyujin, C. phaeocaulis and C. kwangsiensis, respectively, which could be used to discriminate between different Curcumae Radix samples in terms of their species of origin.

\section{Conclusions}

This study developed a UHPLC/Q-TOFMS method coupled with multivariate statistical analysis to discriminate between Curcumae Radix samples from four different Curcumae species, i.e., C. longa, C. wenyujin, $C$. 
a

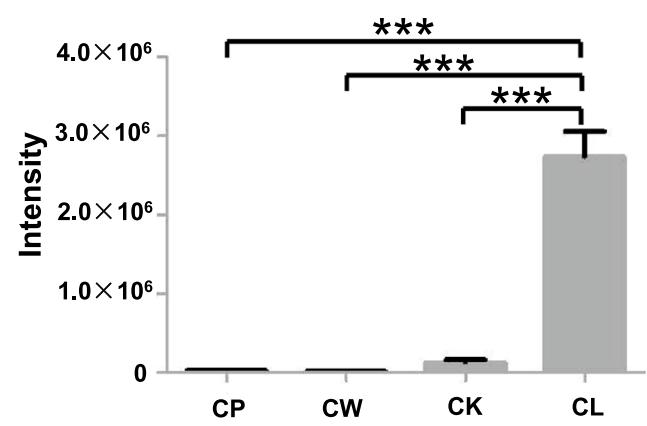

C

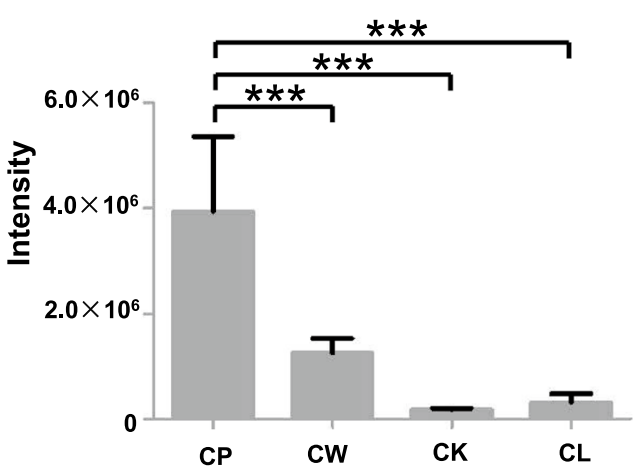

e

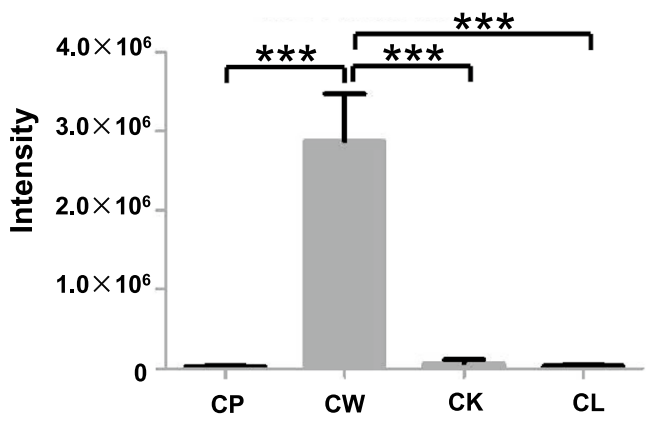

g

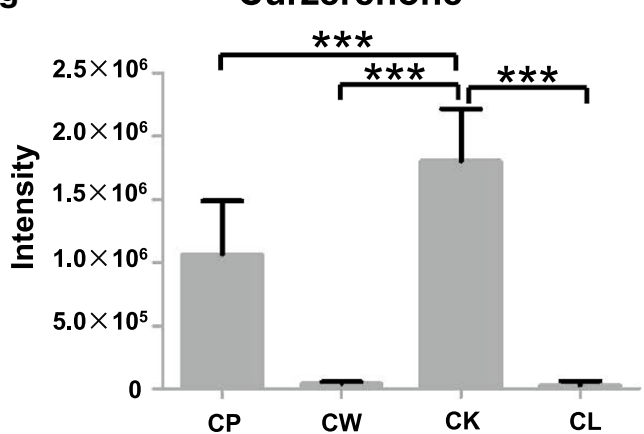

b

Curcumenone

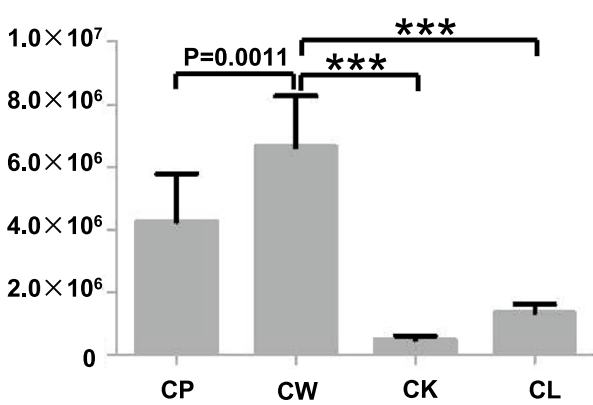

d

Zederone

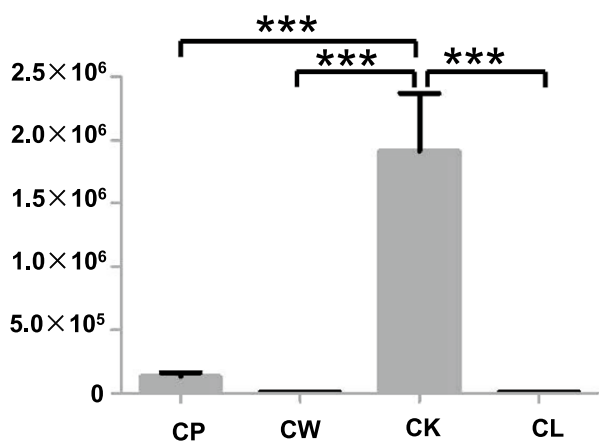

$\mathbf{f}$

Curdione

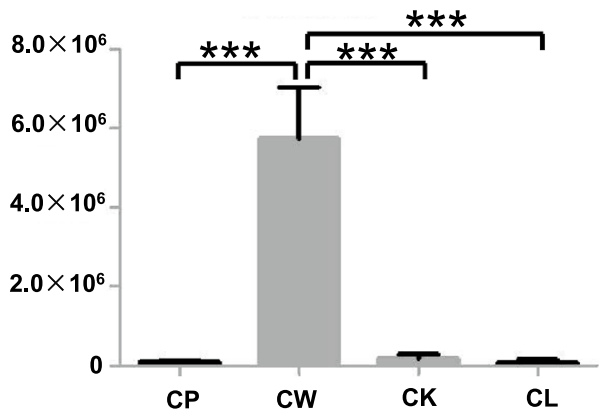

Fig. 5 Relative intensities of the chemical markers in Curcuma Radix derived from four species. (a) curcumin, (b) curcumenone, (c) curcumenol, (d) zederone, (e) neocurdione, (f) curdione and $(\mathbf{g})$ curzerenone. Data represent the mean values $\pm \mathrm{SD}(\mathrm{n}=3)$. ${ }^{* *}$ and ${ }^{* * * *}$ indicate $P<0.01$ and $P<0.0001$, respectively 

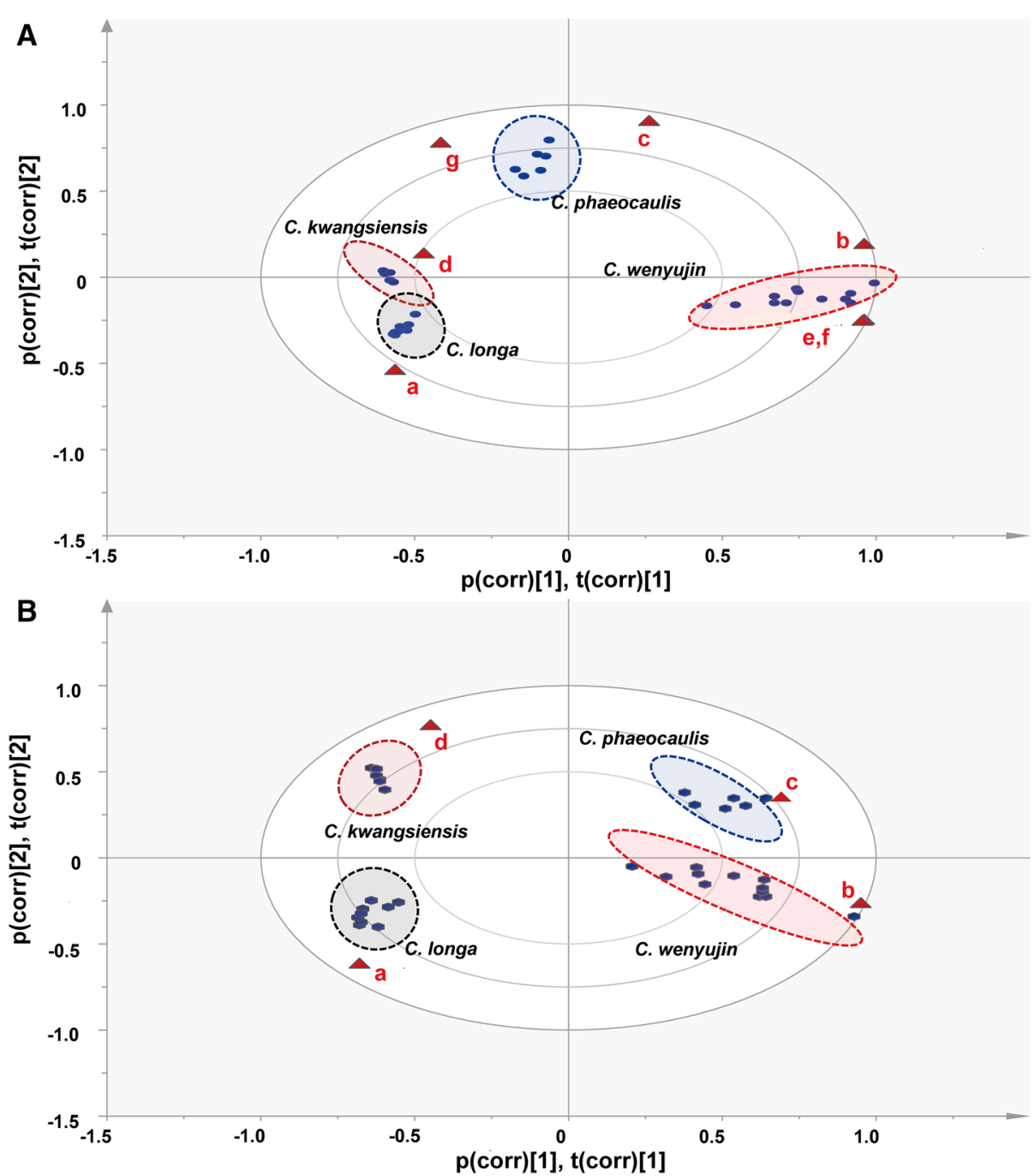

Fig. 6 PCA/bi-plots of the 33 Curcuma Radix samples constructed using $(\mathbf{A})$ seven chemical markers $(\mathbf{a}-\mathbf{g})$ and $(\mathbf{B})$ four unique chemical markers (a-d). (a) curcumin, (b) curcumenone, (c) curcumenol, (d) zederone, (e) neocurdione, (f) curdione and (g) curzerenone

phaeocaulis and C. kwangsiensis. This method was used to identify curcumin (a), curcumenone (b), curcumenol (c) and zederone (d) as unique chemical markers of four Yujin.

\section{Additional file}

Additional file 1. Mass spectra in the positive ionization mode and chemical structures of $(\mathbf{A})$ curcumenol and $(\mathbf{B})$ zederone, $(\mathbf{C})$ neocurdione, (D) curdione and (E) curzerenone.

\section{Abbreviations}

BPI: based peak intensity; CE: capillary electrophoresis; GAP: good agricultural practice; GC-MS: gas chromatography-mass spectrometry; HPLC: highperformance liquid chromatography; TLC: thin layer chromatography; $\mathrm{m} / \mathrm{z}$ : mass-to-charge ratio; OPLS-DA: orthogonal partial least squared discriminant analysis; PCA: principal component analysis; PLE: pressurized liquid extractions; QC: quality control; RSDs: relative standard deviations; $R T$ or $t_{R}$ : retention time;
CMs: chinese medicines; UPLC/Q-TOFMS: ultra-performance liquid chromatography-quadrupole time-of-flight mass spectrometry; VIP: variable importance on projection.

\section{Authors' contributions}

JBW conceived and designed the study. XB performed the LC-MS analysis experiment and FL collected the data. FL and JBW wrote the manuscript. FQY, $\mathrm{YJH}$ and $\mathrm{PL}$ revised the manuscript. All authors read and approved the final manuscript.

\section{Author details}

${ }^{1}$ State Key Laboratory of Quality Research in Chinese Medicine, Institute of Chinese Medical Sciences, University of Macau, Macao, People's Republic of China. ${ }^{2}$ Waters Technologies (Shanghai) Ltd., Shanghai, People's Republic of China. ${ }^{3}$ School of Chemistry and Chemical Engineering, Chongqing University, Chongqing 400030, People's Republic of China.

\section{Acknowledgements}

This study was supported by the grants from the Research Committee of the University of Macau (MYRG123-ICMS12 and MYRG111-ICMS13 to JB Wan) and from the Macao Science and Technology Development Fund (010/2013/A1 to JB Wan). 


\section{Competing interests}

Xu Bai is an employee of the Waters Technologies who is the manufacturer of the UHPLC-Q-TOFMS used in this study. Other authors have no competing interests.

Received: 10 August 2014 Accepted: 20 April 2016

Published online: 29 April 2016

\section{References}

1. Chinese Pharmacopoeia commission. Pharmacopoeia of the People's Republic of China. Beijing: China Medical Science Press; 2010.

2. Yi T, Chen HB, Zhao ZZ, Jiang ZH, Cai SQ. Comparative analysis of the major constituents in the traditional Tibetan medicinal plants Saussurea laniceps and S. medusa by LC-DAD-MS. Chromatographia. 2009;70:957-62.

3. Yi T, Chen HB, Zhao ZZ, Yu ZL, Jiang ZH. Comparison of the chemical profiles and anti-platelet aggregation effects of two "Dragon's Blood" drugs used in traditional Chinese medicine. J Ethnopharmacol. 2011;133:796-802.

4. Zhang XJ, Qiu JF, Guo LP, Wang Y, Li P, Yang FQ, Su H, Wan JB. Discrimination of multi-origin chinese herbal medicines using gas chromatography-mass spectrometry-based fatty acid profiling. Molecules. 2013;18:15329-43.

5. Chai XY, Li SL, Li P. Quality evaluation of Flos lonicerae through a simultaneous determination of seven saponins by HPLC with ELSD. J Chromatogr A. 2005;1070:43-8

6. Lu B, Xu L, Yu L, Zhang L. Extract of radix curcumae prevents gastric cancer in rats. Digestion. 2008;77:87-91.

7. Lan FY. Pharmacological activity and clinical application of Yujing. Changchun Zhongyiyao Daxue Xuebao. 2009;25:27-8.

8. Tao W, Xu X, Wang X, Li B, Wang Y, Li Y, Yang L. Network pharmacologybased prediction of the active ingredients and potential targets of Chinese herbal Radix Curcumae formula for application to cardiovascular disease. J Ethnopharmacol. 2013;145:1-10.

9. Nishiyama T. Curcuminoids and Sesquiterpenoids in turmeric(Curcuma longa L.) suppress an increase in blood glucose level in type 2 diabetic KK-Ay Mice. J Agric Food Chem. 2005;53:959-63.

10. Commission CP. Pharmacopoeia of the People's Republic of China edition, In: Yujin. Beijing: China Medical Science Press; 2010. pp. 193-194.

11. Jiang H, Timmermann BN, Gang DR. Use of liquid chromatographyelectrospray ionization tandem mass spectrometry to identify diarylheptanoids in turmeric (Curcuma longa L.) rhizome. J Chromatogr A. 2006;1111:21-31.

12. Shen $Y$, Han C, Chen X, Hou X, Long Z. Simultaneous determination of three Curcuminoids in Curcuma wenyujin Y.H.chen et C.Ling. by liquid chromatography-tandem mass spectrometry combined with pressurized liquid extraction. J Pharm Biomed Anal. 2013;81-82:146-50.

13. Ni Y, Mei M, Kokot S. One- and two-dimensional gas chromatographymass spectrometry and high performance liquid chromatography-diodearray detector fingerprints of complex substances: a comparison of classification performance of similar, complex Rhizoma Curcumae samples with the aid of chemometrics. Anal Chim Acta. 2012;712:37-44.

14. Zhu JJ, An YW, Hu G, Yin GP, Zhang QW, Wang ZM. Simultaneous determination of multiple sesquiterpenes in Curcuma wenyujin herbal medicines and related products with one single reference standard. Molecules. 2013;18:2110-21.

15. An YW, Hu G, Yin GP, Zhu JJ, Zhang QW, Wang ZM, Peng J, Fan B. Quantitative analysis and discrimination of steamed and non-steamed rhizomes of Curcuma wenyujin by GC-MS and HPLC. J Chromatogr Sci. 2013;52:961.
16. Yang FQ, Li SP, Zhao J, Lao SC, Wang YT. Optimization of GC-MS conditions based on resolution and stability of analytes for simultaneous determination of nine sesquiterpenoids in three species of Curcuma rhizomes. J Pharm Biomed Anal. 2007:43:73-82.

17. Zhang JS, Guan J, Yang FQ, Liu HG, Cheng XJ, Li SP. Qualitative and quantitative analysis of four species of Curcuma rhizomes using twice development thin layer chromatography. J Pharm Biomed Anal. 2008;48:1024-8.

18. Lechtenberg M, Quandt B, Nahrstedt A. Quantitative determination of curcuminoids in Curcuma rhizomes and rapid differentiation of Curcuma domestica Val. and Curcuma xanthorrhiza Roxb. by capillary electrophoresis. Phytochem Anal. 2004;15:152-8.

19. Xiang Z, Wang XQ, Cai XJ, Zeng S. Metabolomics study on quality control and discrimination of three curcuma species based on gas chromatograph-mass spectrometry. Phytochem Anal. 2011;22:411-8.

20. Lee EJ, Shaykhutdinov R, Weljie AM, Vogel HJ, Facchini PJ, Park SU, Kim YK, Yang TJ. Quality assessment of ginseng by (1)H NMR metabolite fingerprinting and profiling analysis. J Agric Food Chem. 2009;57:7513-22.

21. Chan EC, Yap SL, Lau AJ, Leow PC, Toh DF, Koh HL. Ultra-performance liquid chromatography/time-of-flight mass spectrometry based metabolomics of raw and steamed Panax notoginseng. Rapid Commun Mass Spectrom. 2007;21:519-28.

22. Toh DF, New LS, Koh HL, Chan EC. Ultra-high performance liquid chromatography/time-of-flight mass spectrometry (UHPLC/TOFMS) for timedependent profiling of raw and steamed Panax notoginseng. J Pharm Biomed Anal. 2010;52:43-50.

23. Yang SO, Shin YS, Hyun SH, Cho S, Bang KH, Lee D, Choi SP, Choi HK. NMRbased metabolic profiling and differentiation of ginseng roots according to cultivation ages. J Pharm Biomed Anal. 2012;58:19-26.

24. Sun J, Chen P. Differentiation of Panax quinquefolius grown in the USA and China using LC/MS-based chromatographic fingerprinting and chemometric approaches. Anal Bioanal Chem. 2011;399:1877-89.

25. Baldovini N, Tomi F, Casanova J. Identification and quantitative determination of furanodiene, a heat-sensitive compound, in essential oil by 13C-NMR. Phytochem Anal. 2001;12:58-63.

26. Ishii H, Tozyo T, Nakamura M, Takeda K. Components of the root of lindera strychnifolia Vill—XIII: structure of isogemafurene and lineroxide. Tetrahedron. 1968;24:625-31.

27. Zhonghua Bencao Editorial Committee Chinese State Administration of TCM, Zhonghua Bencao. Shanghai: Shanghai Scientific and Technical Press; 1999.

28. Yang FQ, Wang YT, Li SP. Simultaneous determination of 11 characteristic components in three species of Curcuma rhizomes using pressurized liquid extraction and high-performance liquid chromatography. J Chromatogr A. 2006;1134:226-31.

29. Wan JB, Bai X, Cai XJ, Rao Y, Wang YS, Wang YT. Chemical differentiation of Da-Cheng-Qi-Tang, a Chinese medicine formula, prepared by traditional and modern decoction methods using UPLC/Q-TOFMS-based metabolomics approach. J Pharm Biomed Anal. 2013;83:34-42.

30. He XGLLZ, Lian LZ, Lindenmaier M. Liquid chromatography-electrospray mass spectrometric analysis of curcuminoids and sesquiterpenoids in turmeric (Curcuma longa). J Chromatogr A. 1998;818:127-32.

31. Yang FQ, Wang HK, Chen H, Chen JD, Xia ZN. Fractionation of volatile constituents from Curcuma rhizome by preparative gas chromatography. J Autom Methods Manag Chem. 2011;2011:942467.

32. Qin NY, Yang FQ, Wang YT, Li SP. Quantitative determination of eight components in rhizome (Jianghuang) and tuberous root (Yujin) of Curcuma longa using pressurized liquid extraction and gas chromatography-mass spectrometry. J Pharm Biomed Anal. 2007:43:486-92. 